\title{
Analysing microbiome intervention design studies: Comparison of alternative multivariate statistical methods
}

\author{
Maryia Khomich $\oplus^{1,2 *}$, Ingrid Måge ${ }^{3 *}$, Ida Rud ${ }^{1}$, Ingunn Berget ${ }^{3}$ \\ 1 Division of Food Science, Department of Food Safety and Quality, Nofima - Norwegian Institute of Food, \\ Fisheries and Aquaculture Research, Ås, Norway, 2 Department of Clinical Science, University of Bergen, \\ Bergen, Norway, 3 Division of Food Science, Department of Raw Materials and Process Optimisation, \\ Nofima - Norwegian Institute of Food, Fisheries and Aquaculture Research, Ås, Norway \\ * marykhomich@gmail.com, maryia.khomich@uib.no (MK); ingrid.mage@nofima.no (IM)
}

\section{G open Access}

Citation: Khomich M, Måge I, Rud I, Berget I (2021) Analysing microbiome intervention design studies: Comparison of alternative multivariate statistical methods. PLOS ONE 16(11): e0259973. https://doi.org/10.1371/journal.pone.0259973

Editor: Lingling An, University of Arizona, UNITED STATES

Received: March 10, 2021

Accepted: October 30, 2021

Published: November 18, 2021

Peer Review History: PLOS recognizes the benefits of transparency in the peer review process; therefore, we enable the publication of all of the content of peer review and author responses alongside final, published articles. The editorial history of this article is available here: https://doi.org/10.1371/journal.pone.0259973

Copyright: @ 2021 Khomich et al. This is an open access article distributed under the terms of the Creative Commons Attribution License, which permits unrestricted use, distribution, and reproduction in any medium, provided the original author and source are credited.

Data Availability Statement: All relevant data are within the paper and its Supporting information files.

Funding: This work was funded by Nofima Norwegian Institute of Food, Fisheries and

\section{Abstract}

The diet plays a major role in shaping gut microbiome composition and function in both humans and animals, and dietary intervention trials are often used to investigate and understand these effects. A plethora of statistical methods for analysing the differential abundance of microbial taxa exists, and new methods are constantly being developed, but there is a lack of benchmarking studies and clear consensus on the best multivariate statistical practices. This makes it hard for a biologist to decide which method to use. We compared the outcomes of generic multivariate ANOVA (ASCA and FFMANOVA) against statistical methods commonly used for community analyses (PERMANOVA and SIMPER) and methods designed for analysis of count data from high-throughput sequencing experiments (ALDEx2, ANCOM and DESeq2). The comparison is based on both simulated data and five published dietary intervention trials representing different subjects and study designs. We found that the methods testing differences at the community level were in agreement regarding both effect size and statistical significance. However, the methods that provided ranking and identification of differentially abundant operational taxonomic units (OTUs) gave incongruent results, implying that the choice of method is likely to influence the biological interpretations. The generic multivariate ANOVA tools have the flexibility needed for analysing multifactorial experiments and provide outputs at both the community and OTU levels; good performance in the simulation studies suggests that these statistical tools are also suitable for microbiome data sets.

\section{Introduction}

The microbiome has emerged as an important link to health and disease [1]. Microbiome analysis methods are rapidly advancing, in particular in areas such as compositional data analysis, multi-omics and data integration [2,3]. A clear understanding of the type of data being analysed is crucial, given the growing number of studies uncovering the key role of microbiome, its composition and functions following diet intervention or medical treatment [4]. At present, analysis of complex microbial data benefits from adapting the multivariate statistical 
Aquaculture Research and Foundation for Research Levy on Agricultural Products (Research Council of Norway projects No. 262306, 262308, 314111 and 314743$)$. The funding sponsors had no role in the design of the study, the collection, analyses and interpretation of data, in the writing of the manuscript or in the decision to distribute the results.

Competing interests: The authors declare no competing interests.

Abbreviations: ALDEX2, ANOVA-like differential
expression tool for high-throughput sequencing
data; ANCOM, Analysis of composition of
microbiomes; ANOSIM, Analysis of similarities;
ANOVA, Analysis of variance; ASCA, ANOVA-
simultaneous component analysis; DESeq2,
Differential gene expression analysis based on the
negative binomial distribution; FDR, False
discovery rate; FFMANOVA, Fifty-fifty multivariate
ANOVA; OTU, Operational taxonomic unit;
PERMANOVA, Permutational multivariate analysis
of variance; PLS-DA, Partial least squares
discriminant analysis; SIMPER, Similarity
percentage.
percentage. toolbox from ecology and environmental sciences, and a proper choice of statistical tools is becoming increasingly important [5-7]. However, a lack of benchmarking studies and clear consensus on the best multivariate statistical practices make comparisons across microbiome data sets difficult $[2,8]$. New methods are often tested by simulation studies, but there is always a concern that simulations can be biased towards the tested statistical model and cannot mimic the complexity of real microbiome data $[6,9]$. Moreover, newly introduced tools are often optimised, whereas the comparison of several statistical methods implies the use of standard or default parameters [6, 9]. It is therefore of interest to compare existing methods on real data sets of different complexity, in addition to simulation studies, to better understand how choice of method affects the results.

Different statistical methods have different properties, and the choice of method should depend on the scientific question, experimental design, data characteristics and expected relationships among the variables. Furthermore, the choice of method is often biased by the research groups' tradition and familiarity with specific "toolboxes". Main differences between existing statistical approaches for analysing microbiome data are related to: (1) explorative versus confirmative; (2) univariate versus multivariate; (3) parametric versus nonparametric; (4) linear versus nonlinear; (5) compositional versus non-compositional; (6) distance-based versus count/abundance-based; and (7) incorporating phylogenetic information into the analysis or not [10-12]. Here, we explore statistical methods for analysing microbiome data from designed experiments with a focus on dietary intervention trials. In contrast to observational studies, these are usually small in sample size but performed in (semi)-controlled environments and tailored to a specific research hypothesis. The studies often include multiple experimental factors, possibly with more than two levels, and it is therefore natural to turn to analysis of variance (ANOVA)-like methods. Notably, most published analytical tools in microbiome research are essentially univariate [6], which led us to the conclusion that comparison of alternative multivariate statistical tools is sorely missing. From a biologist's point of view, it is also important that the methods are easy to interpret, both at the multivariate (microbial community) and univariate (microbial taxa or operational taxonomic units, OTUs) levels (see Fig 1).

\section{Distance-based methods}

The distance-based methods are multivariate since multiple variables (microbial OTUs) are used to calculate pairwise distances between samples. Among distance-based methods, permutational multivariate analysis of variance (PERMANOVA) is the most widely used and more powerful than the analysis of similarities (ANOSIM) to detect changes in community structure [13-15]. Both methods may be implemented with any dissimilarity metric. Among abundance-based beta diversity indices, Bray-Curtis is the most common choice for count data [16, 17]. The most widely applied phylogenetic beta diversity indices are UniFrac-type metrics [17-19]. However, UniFrac is unsuitable as a distance metric for studies with a small sample size, which is usually the case for dietary intervention trials [20, 21]. Both PERMANOVA and ANOSIM test differences at the community level but do not provide any information at the OTU level. Similarity percentage analysis (SIMPER) works at the univariate level by computing the relative contribution of each analysed microbial taxon (i.e. OTU) to the overall average Bray-Curtis dissimilarities by pairwise comparison of two or more groups [15]. To the best of our knowledge, no such method exists for the other distance metrics.

Distance-based methods have their strengths and weaknesses that are important to account for beforehand. ANOSIM cannot deal with multifactorial designs, and both ANOSIM and PERMANOVA may have problems detecting differences unless they are present in taxa with 


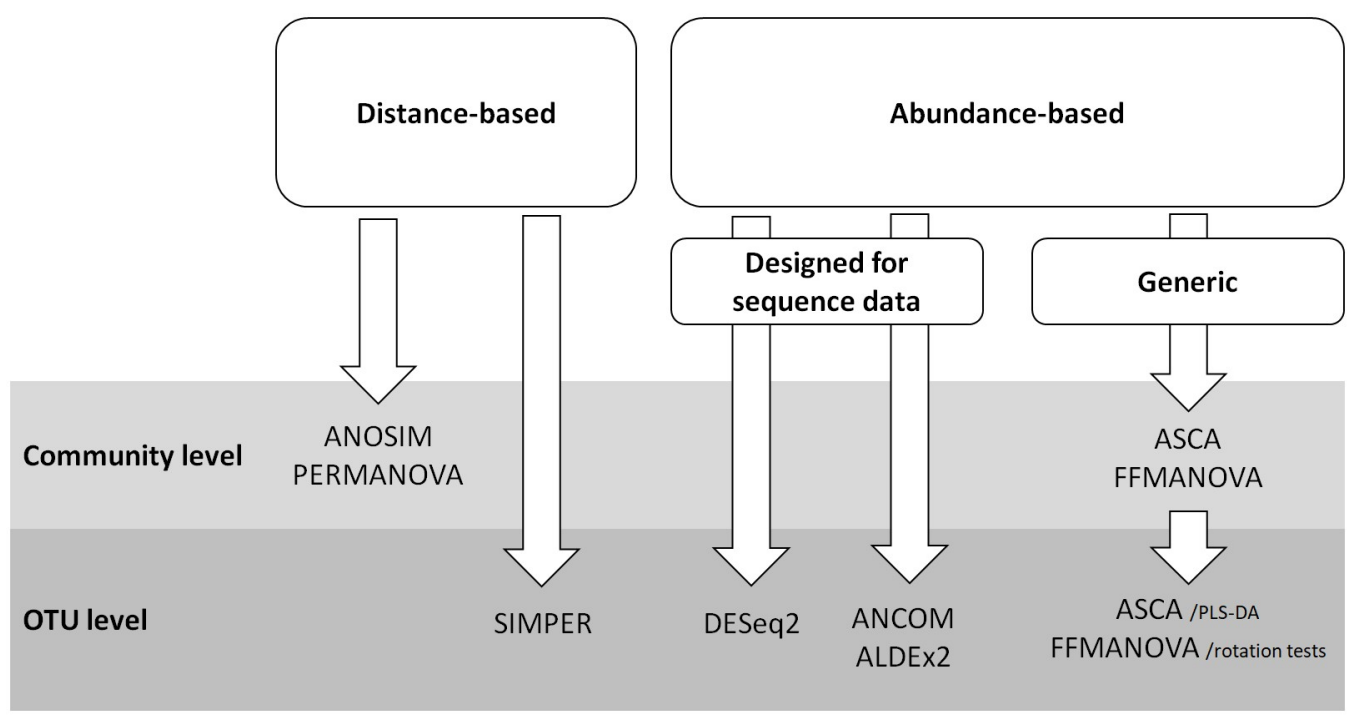

Fig 1. A diagram of statistical methods used in the study.

https://doi.org/10.1371/journal.pone.0259973.g001

high variability [22]. Newer methods aimed at assigning more interpretable effect sizes are under constant development $[6,23]$. For example, the more flexible PERMANOVA-S is an extension to existing distance-based methods that can adjust for covariates and simultaneously incorporate multiple distance metrics [24]. However, these methods do not consider covariance/correlation between microbial species, and they encounter significant power loss if all microbial species are used for distance calculations [25].

\section{Abundance-based methods}

The abundance-based methods can be either univariate (analysing each OTU individually) or multivariate (focusing on covariance structure between OTUs). There are two main approaches to deal with the special nature of abundance data: (1) application of methods that consider the distribution of count data or (2) compositional data analysis (CoDa) based on $\log$-ratio transformed count data [26, 27]. Statistical methods designed for high-throughput sequencing data are ANOVA-like differential expression analysis (ALDEx2) [26], analysis of composition of microbiomes (ANCOM) [28], edgeR [29] and DESeq/DESeq2 [30]. edgeR and DESeq2 model count data directly using generalized linear models with the negative binomial distribution and the logistic link, respectively, whereas ALDEx2 and ANCOM use the log-ratio transformation prior to univariate assessment of statistical significance for individual OTUs. DESeq2 and edgeR are based on the same modelling approach but differ in normalisation, outlier handling, and other adjustable parameters; these methods had similar performance in simulation studies [30]. Thus, we decided to include only one of the methods-DESeq2-because differences between DESeq2 and edgeR are at a different conceptual level rather than the other methods discussed. ALDEx2 uses a Dirichlet-multinomial probability distribution to estimate abundances from count data and calculates the false discovery rate (FDR) based on Monte Carlo simulations (see Fig 3 in [26] for details). In ANCOM, the compositional nature of the data is considered by testing the log-ratio for all pairs of OTUs, and then counting the number of tests where the log-ratio is significantly different from zero. This number (W-stat) can be used to obtain a ranking of OTUs most likely to differ between the groups. The newly published ANCOM-BC corrects the bias induced by differences in sampling fractions and 
provides p-values and confidence intervals for the differential abundance of each OTU [31]. The FDR and power were shown to be similar for both ANCOM and ANCOM-BC, and therefore we limit the present study to ANCOM.

The drawback of univariate methods is that they treat all taxa as independent variables without considering the covariance between the OTUs. Such methods may fail to detect community-level differences [32]. A classical generalisation of ANOVA to multiple variables (MANOVA) cannot be used when the number of variables exceeds the number of samples, as it suffers from the problem of a singularity of covariance matrices and assumptions that are not fulfilled [33,34]. Novel statistical ANOVA-like methods include fifty-fifty multivariate analysis of variance (FFMANOVA) [35] and ANOVA-simultaneous component analysis (ASCA) [33]. Both methods are based on principal component analysis (PCA), and they can handle multiple collinear responses. In FFMANOVA, the multivariate effects are estimated by a modified variant of classical MANOVA, and OTU-level p-values are obtained by rotation tests which adjust the p-values for multiple testing [36]. For ASCA, the multivariate effects are calculated from combined sums-of-squares from all OTUs, and significance is assessed by permutation testing. ASCA also provides scores and loadings related to each experimental factor, which can be visualised in the same way as for PCA to better understand covariance patterns within the data. The contribution of each OTU can be quantified by the loadings or by partial least squares discriminant analysis (PLS-DA) for pairwise comparisons. ASCA has recently gained popularity in metabolomics [37-39], and both ASCA and FFMANOVA have successfully been applied to microbiome data [40-44].

Linear discriminant analysis effect size (LEfSe) is a stepwise approach that combines univariate analysis with multivariate discriminant analysis [45]. LEfSe has found wide application in microbiome research due to its easy to-use-and-interpret visualization [46, 47], but it is not adapted to experimental designs with several multilevel factors and is therefore not considered in this study.

\section{Method comparison}

An overview of the different methods compared in this study is given in Fig 1 and Table 1. ANOSIM and PERMANOVA provide results only at the community level, while SIMPER, DESeq2, ANCOM and ALDEx2 report results for single OTUs. ASCA and FFMANOVA are generic methods and the only methods that provide results at both the community and OTU level.

The aim of the method comparison was to investigate how different strategies for statistical modelling affect biological inference. At the community level, methods were compared with respect to effect sizes (expressed as percentage of explained variance) and corresponding p-values. At the OTU level, comparison of methods is complex because some methods provide results for an omnibus test of differences between factor levels (FFMANOVA and ANCOM), whereas the other methods provide ranking for specific pairwise comparisons (ASCA and PLS-DA, SIMPER) or contrasts/model coefficients (ALDEx2 and DESeq2). Even so, a biologist will make inferences based on the output provided by the chosen method, and in this context, it is relevant to compare the ranking statistics although the tests are not the same. In our study, the ranking of OTUs was compared by Spearman's rank correlation and by investigation of scatterplots between the different ranking metrics. For the simulated data, where we know which OTUs are differentially abundant, True Positive Rate (TPR) and True Negative Rate (TNR) were also evaluated.

We focused exclusively on designed experiments, which are usually smaller in sample size and are more controlled in contrast to observational studies. We used five published data sets 
Table 1. An overview of statistical methods and their properties.

\begin{tabular}{|c|c|c|c|c|c|c|c|c|}
\hline Method & Method name & $\begin{array}{c}\text { Number of } \\
\text { experimental } \\
\text { factors allowed }\end{array}$ & Parametric & Multivariate & Univariate & $\begin{array}{c}\text { Provides output } \\
\text { at community } \\
\text { level }\end{array}$ & $\begin{array}{l}\text { Statistics for } \\
\text { ranking OTUs }\end{array}$ & Reference \\
\hline ALDEx2 & $\begin{array}{l}\text { ANOVA-like differential } \\
\text { expression tool for high- } \\
\text { throughput sequencing data }\end{array}$ & any & yes & no & yes & no & $\begin{array}{l}\text { p-values or effect } \\
\text { sizes }\end{array}$ & [26] \\
\hline ANCOM & $\begin{array}{l}\text { Analysis of composition of } \\
\text { microbiomes }\end{array}$ & $\begin{array}{l}\text { main factor } \\
+ \text { covariates }\end{array}$ & yes & no & yes & no & $\begin{array}{l}\mathrm{W} \text {-stat for the } \\
\text { main variable }\end{array}$ & [28] \\
\hline ANOSIM & Analysis of similarities & one & no & yes & no & yes & no & [15] \\
\hline ASCA & $\begin{array}{l}\text { ANOVA-simultaneous } \\
\text { component analysis }\end{array}$ & any & yes & yes & no & yes & $\begin{array}{l}\text { loadings or } \\
\text { PLS-DA regression } \\
\text { coefficients }\end{array}$ & [33] \\
\hline DESeq2 & $\begin{array}{l}\text { Differential gene expression } \\
\text { analysis based on the } \\
\text { negative binomial } \\
\text { distribution }\end{array}$ & any & yes (GLM) & no & yes & no & $\begin{array}{l}\text { p-values or effect } \\
\text { sizes (coefficients) }\end{array}$ & {$[30]$} \\
\hline FFMANOVA & $\begin{array}{c}\text { Fifty-fifty multivariate } \\
\text { ANOVA }\end{array}$ & any & yes & yes & $\begin{array}{l}\text { yes (rotation } \\
\text { tests) }\end{array}$ & yes & p-values & [35] \\
\hline PERMANOVA & $\begin{array}{l}\text { Permutational multivariate } \\
\text { analysis of variance }\end{array}$ & any & no & yes & no & yes & no & [14] \\
\hline SIMPER & Similarity percentage & $\begin{array}{l}\text { two-group } \\
\text { comparison }\end{array}$ & no & yes & no & no & $\begin{array}{l}\text { permutation p- } \\
\text { values }\end{array}$ & [15] \\
\hline
\end{tabular}

ANOVA — analysis of variance; GLM - generalized linear model; OTU—operational taxonomic unit; PLS-DA—partial least squares discriminant analysis.

as a basis for the comparisons (S1 Table). The following criteria for studies to be included were considered: (1) at least two-factorial experimental design with a minimum of two-factor levels; (2) either human or animal gut microbiome surveys; and (3) a taxonomic assignment at the OTU level reported. Diet is the main factor of interest in all five studies, and we restricted our comparisons to this factor.

The simulated data were based on data set 1 (S1 Table) using the same study design and OTU counts as a starting point. Four different scenarios were simulated to investigate how the methods perform in situations with varying effect sizes and different numbers of differentially abundant OTUs. In all scenarios, one of the diet levels was manipulated to be significantly different from the others, and there was no effect of the second experimental factor (dose). See Methods section for further details.

\section{Results}

\section{Community level}

Four of the methods can be used to test the association between diet and the overall microbiome composition, the distance-based ANOSIM and PERMANOVA, and abundance-based ASCA and FFMANOVA. The results for each of the four simulated scenarios are shown in Fig 2, and the results across real data sets are summarised in Table 2.

Simulated data. As expected, the multivariate effect size (explained variance) is lowest (around 5\%) for the "Few-Low" scenario and highest (30-35\%) for the "Many-High" scenario. The multivariate effect was significant in $100 \%$ of the simulations in three scenarios with the highest effect size. For the "Few-Low" scenario, FFMANOVA performed best by detecting the effect in $80 \%$ of the data sets. For the "Many" simulations explained variance was slightly higher with FFMANOVA than with ASCA and PERMANOVA, whereas for the "Few-High" simulations the opposite trend was observed. ANOSIM was less consistent than the other 


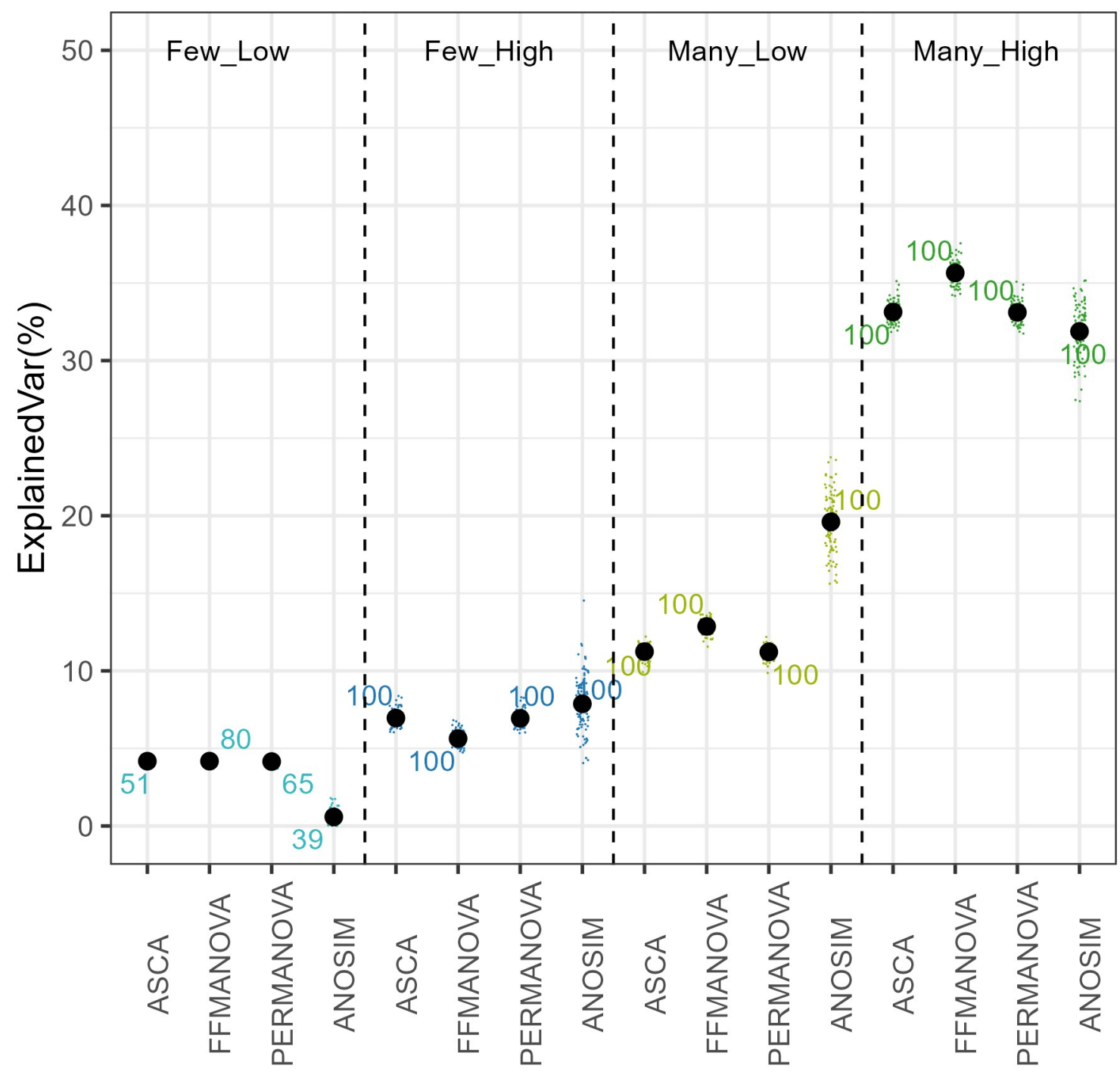

Fig 2. Explained variance for simulated data and the relative number of simulations where the simulated effect was detected. Numbers indicate the percentage of simulated data sets where p-value was significant.

https://doi.org/10.1371/journal.pone.0259973.g002

methods, with higher within-scenario variation and higher differences in effect size between the two "Low" scenarios.

Data set 1. The effect of dietary fibres inulin (IN), cellulose (CE) or brewers spent grain (BSG) on the overall caecal microbiota composition in mice from a study by Moen et al. [42] accounted for $34-37 \%$ of the explained variance according to the FFMANOVA, ASCA and PERMANOVA. In general, the three methods produced similar results, with slightly smaller p-values by FFMANOVA and ASCA.

Data set 2. Lai et al. [48] investigated the effect of diet (the main variable), exercise and their interaction on the overall faecal microbiota in sedentary and exercised mice fed high fat or normal fat diet (four groups in total). Similarly, all tested methods, except ANOSIM, produced congruent results (effect of diet 27-31\%), with smaller p-values by FFMANOVA and ASCA. 
Table 2. Community-level method comparison across five experimental data sets.

\begin{tabular}{|c|c|c|c|c|c|c|c|c|c|}
\hline \multirow{2}{*}{$\begin{array}{l}\text { Factor/ } \\
\text { predictor }\end{array}$} & \multicolumn{2}{|l|}{ FFMANOVA $^{\text {clr }}$} & \multicolumn{2}{|l|}{ ASCA $^{\text {clr }}$} & \multicolumn{2}{|l|}{ PERMANOVA $^{\mathrm{clr}}$} & \multicolumn{2}{|l|}{ ANOSIM $^{3, \text { clr }}$} & \multirow{2}{*}{$\begin{array}{c}\text { Factor/ } \\
\text { predictor }\end{array}$} \\
\hline & $\begin{array}{l}\text { Effect size (explained } \\
\text { variance), \% }\end{array}$ & p-value ${ }^{1}$ & \begin{tabular}{|c|} 
Effect size (explained \\
variance), \%
\end{tabular} & $\begin{array}{c}\text { p- } \\
\text { value }^{2}\end{array}$ & $\begin{array}{c}\text { Effect size (explained } \\
\text { variance), \% }\end{array}$ & $\begin{array}{c}\text { p- } \\
\text { value }^{2}\end{array}$ & $\begin{array}{c}\text { Effect size (explained } \\
\text { variance), \% }\end{array}$ & $\begin{array}{c}\text { p- } \\
\text { value }^{2}\end{array}$ & \\
\hline \multicolumn{7}{|c|}{ Moen et al., 2016 (data set 1$)$} & \multicolumn{3}{|c|}{ Moen et al., 2016 (data set 1) } \\
\hline \multicolumn{7}{|c|}{ Model: OTU fiber dose } & \multicolumn{3}{|c|}{ Model: OTU fiber_dose } \\
\hline fiber & 34.31 & $<0.001$ & 37.39 & $\begin{array}{c}< \\
0.001 \\
\end{array}$ & 37.26 & 0.001 & \multirow[t]{3}{*}{82.19} & \multirow[t]{3}{*}{0.001} & fiber \\
\hline dose & 3.96 & $<0.001$ & 4.14 & $\begin{array}{c}< \\
0.001\end{array}$ & 4.12 & 0.001 & & & dose \\
\hline fiber:dose & 5.85 & $<0.001$ & 5.99 & $\begin{array}{c}< \\
0.001\end{array}$ & 5.96 & 0.002 & & & fiber:dose \\
\hline residuals & 55.75 & & 52.69 & & 52.67 & & 17.81 & & residuals \\
\hline \multicolumn{7}{|c|}{ Lai et al., 2018 (data set 2) } & \multicolumn{3}{|c|}{ Lai et al., 2018 (data set 2) } \\
\hline \multicolumn{7}{|c|}{ Model: OTU diet ${ }^{*}$ exercise } & \multicolumn{3}{|c|}{ Model: OTU diet_exercise } \\
\hline diet & 27.31 & $<0.001$ & 30.50 & $\begin{array}{c}< \\
0.001 \\
\end{array}$ & 30.85 & 0.001 & \multirow[t]{3}{*}{99.11} & \multirow[t]{3}{*}{0.001} & diet \\
\hline exercise & 12.18 & $<0.001$ & 14.08 & $\begin{array}{c}< \\
0.001\end{array}$ & 13.99 & 0.001 & & & exercise \\
\hline diet:exercise & 7.93 & $<0.001$ & 7.51 & $\begin{array}{c}< \\
0.001 \\
\end{array}$ & 7.47 & 0.002 & & & diet:exercise \\
\hline residuals & 52.31 & & 47.74 & & 47.70 & & 0.89 & & residuals \\
\hline \multicolumn{7}{|c|}{ Le Sciellour et al., 2018 (data set 3) } & \multicolumn{3}{|c|}{ Le Sciellour et al., 2018 (data set 3) } \\
\hline \multicolumn{7}{|c|}{ Model: OTU $\sim \operatorname{diet}^{*}$ period + subject } & \multicolumn{3}{|c|}{ Model: OTU diet_period } \\
\hline diet & 1.78 & $<0.001$ & 1.99 & $\begin{array}{c}< \\
0.001\end{array}$ & 2.14 & 0.001 & \multirow[t]{3}{*}{13.33} & \multirow[t]{3}{*}{0.001} & diet \\
\hline period & 3.29 & $<0.001$ & 3.45 & $\begin{array}{c}< \\
0.001 \\
\end{array}$ & 3.51 & 0.001 & & & period \\
\hline diet:period & 1.32 & $<0.001$ & 1.41 & 0.006 & 1.36 & 0.009 & & & diet:period \\
\hline subject & 26.53 & $<0.001$ & 26.42 & 0.073 & 27.28 & 0.046 & \multirow[t]{2}{*}{86.67} & & subject \\
\hline residuals & 66.13 & & 65.71 & & 65.71 & & & & residuals \\
\hline \multicolumn{7}{|c|}{ Wang et al., 2016 (data set 4) } & \multicolumn{3}{|c|}{ Wang et al., 2016 (data set 4) } \\
\hline \multicolumn{7}{|c|}{ Model: OTU $\sim$ diet + time + diet:time + subject } & Model: OTL & J diet_t & ime \\
\hline diet & 2.49 & 0.067 & 2.11 & 0.038 & 2.27 & 0.036 & 4.32 & 0.143 & diet \\
\hline time & 2.00 & 0.007 & 1.96 & 0.082 & 1.77 & 0.246 & & & time \\
\hline diet:time & 5.15 & 0.824 & 4.41 & 0.777 & 4.45 & 0.701 & & & diet:time \\
\hline subject & 50.44 & $<0.001$ & 54.23 & $\begin{array}{c}< \\
0.001\end{array}$ & 64.30 & 0.001 & 95.68 & & subject \\
\hline residuals & 31.23 & & 27.25 & & 27.21 & & & & residuals \\
\hline & & Birkelan & nd et al., 2020 (data set & & & & Birkeland et al., & $2020(\mathrm{da}$ & ta set 5) \\
\hline & & Model: OT & $\mathrm{U} \sim$ treatment:day + su & ject & & & Model: OTU & treatme & it_day \\
\hline $\begin{array}{c}\text { treatment: } \\
\text { day }\end{array}$ & 1.75 & $<0.001$ & 1.27 & 0.107 & 1.27 & 0.132 & -0.03 & 0.998 & $\begin{array}{l}\text { treatment: } \\
\text { day }\end{array}$ \\
\hline subject & 69.38 & $<0.001$ & 73.85 & 0 & 73.85 & 0.001 & & & subject \\
\hline residuals & 28.88 & & 24.87 & & 24.87 & & & & residuals \\
\hline
\end{tabular}

Distance-based ANOSIM and PERMANOVA and abundance-based ASCA and FFMANOVA were compared with respect to effect sizes (expressed as percentage of explained variance) and corresponding p-values.

${ }^{1}$ based on the 50-50 F-test, 999 permutations.

${ }^{2}$ based on 999 permutations.

${ }^{3}$ based on a combined factor with no interaction in the model (limitation of ANOSIM).

${ }^{\mathrm{clr}}$ centred log-ratio transformed data as input. 
Data set 3. In a longitudinal study by Le Sciellour et al. [49] the authors tested the effect of dietary fibre content on faecal microbiota in growing-finishing pigs fed alternately a low-fibre and a high-fibre diet during four successive 3-week periods. In this survey, the effect of diet was small (2\%) compared to the effect of diet in data sets 1 and 2. Similarly, FFMANOVA and ASCA reported slightly lower p-values than PERMANOVA, but all three methods agreed on the effect of diet.

Data set 4. In a longitudinal study by Wang et al. [50] the objectives were to determine the impact of beta glucan on the composition of faecal microbiota in mildly hypercholesterolemic individuals. The individuals received for 5 weeks either a treatment containing $3 \mathrm{~g}$ high molecular weight (HMW), $3 \mathrm{~g}$ low molecular weight (LMW), $5 \mathrm{~g}$ LMW barley beta glucan or wheat and rice (control group) [50]. The effect of diet accounted for $\sim 2 \%$ of the explained variance reported by FFMANOVA, ASCA and PERMANOVA. Diet was significant on a 5\% level for PERMANOVA and ASCA ( $p=0.036$ and $p=0.038$, respectively) and on a $10 \%$ level for FFMANOVA $(\mathrm{p}=0.067)$. However, different conclusions were drawn with respect to time where significant result at the community level was obtained only for FFMANOVA $(\mathrm{p}=0.007)$.

Data set 5. Birkeland et al. [44] assessed the effect of prebiotic fibres or a control supplement on faecal microbiota composition in human subjects with type two diabetes. The interaction effect of treatment and day accounted for $1-2 \%$ of the explained variance according to the FFMANOVA, ASCA and PERMANOVA. Significant results at the community level were obtained only for FFMANOVA $(\mathrm{p}<0.001)$.

To summarise, PERMANOVA, FFMANOVA and ASCA gave almost identical results regarding effect sizes and statistical significance across studies (Table 2). They all revealed that there was a considerable difference in effect sizes of the main factor of interest (diet) between animal (2-37\%) and human (1-2\%) dietary interventions, with effect sizes being very small in human studies. In addition, three of the studies had crossover designs allowing for estimation of interindividual variation. This variation was considerably higher for trials involving human subjects (54-74\%) compared to the animal study (26\%). ANOSIM provided the most different results and was not able to reveal the same biological insights since the multifactorial nature of the studies cannot be taken into consideration by this approach.

\section{OTU level}

Six of the methods, namely SIMPER, ASCA, FFMANOVA, ANCOM, ALDEx2 and DESeq2 can be used to make biological inferences for individual OTUs. The methods give different outputs which can be used to identify differentially abundant OTUs and/or rank the OTUs according to effect sizes (see Methods for details).

Simulated data. The True Positive Rate (TPR) for the four simulated scenarios is shown in Fig 3. The True Negative Rate (TNR) was close to $100 \%$ for all methods and is therefore not shown. ASCA provided the overall best results in terms of the TPR in all four scenarios. FFMANOVA, SIMPER and ANCOM were all highly sensitive in the scenario with few significant OTUs and a high effect size ("Few-High"). FFMANOVA was also highly sensitive in the scenario with many significant OTUs and a high effect size ("Many-High"); SIMPER and ANCOM performed best in the scenario with few significant OTUs with a lower effect size ("Few-Low"). Both ALDEx2 and DESeq2 detected very few OTUs in any of the scenarios and therefore had very low TPR.

Experimental data. Summary tables and scatterplots comparing ranking for different methods on the experimental data sets are given as S1 File and S1 Fig, respectively. The 


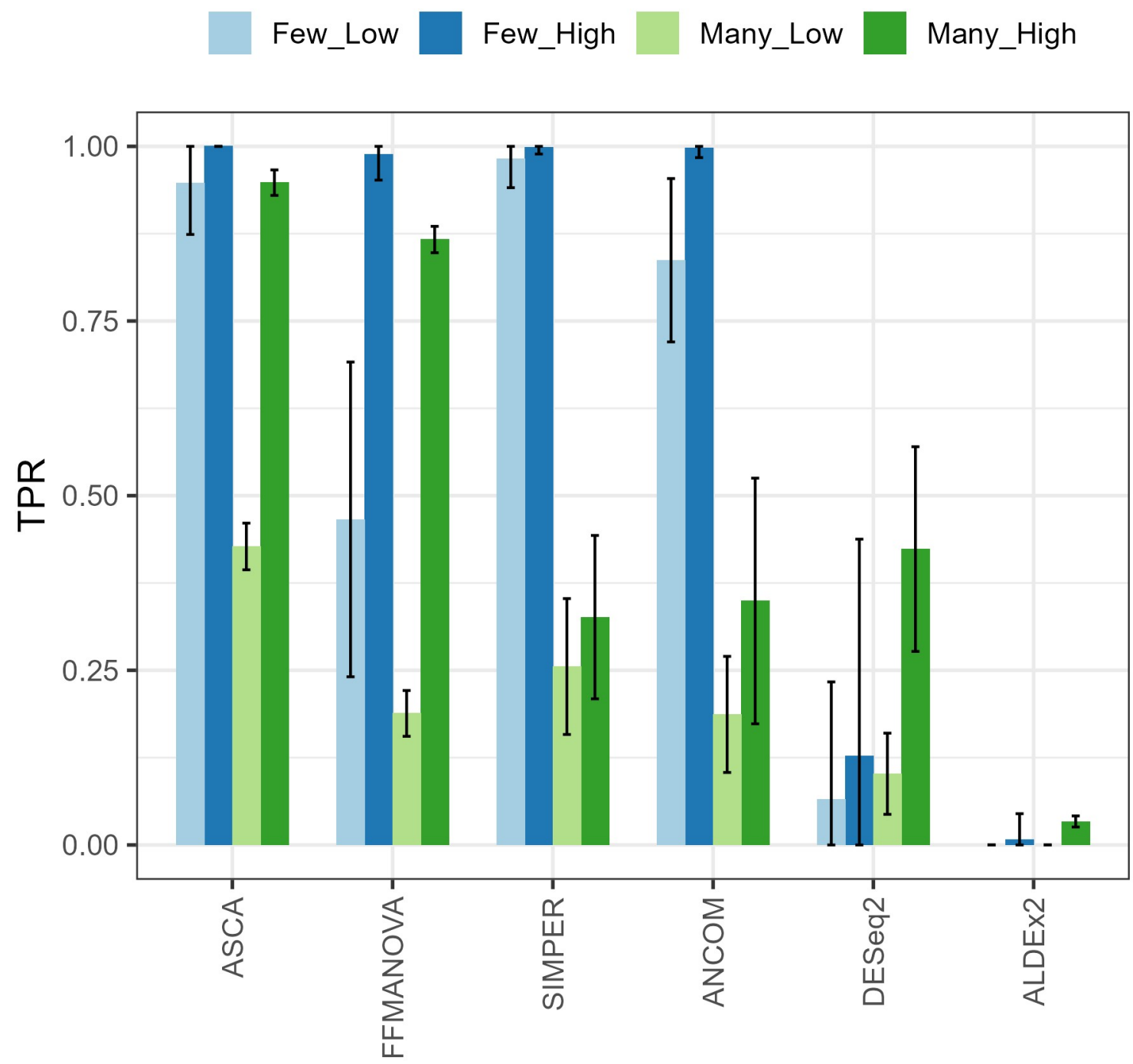

Fig 3. Sensitivity (True Positive Rate) for the four scenarios in the simulation study.

https://doi.org/10.1371/journal.pone.0259973.g003

number of significant OTUs detected by the methods is summarised in S2 Table. As expected, many significant OTUs were discovered in the studies with large multivariate effect sizes (data sets Moen and Lai), whereas few OTUs were found in the studies with low multivariate effect sizes. The highest number of significant OTUs was identified by FFMANOVA, with almost twice the numbers detected by ALDEx2. ANCOM differed considerably between the study designs. ASCA recovered fewer OTUs than the other methods for the Moen data set, but more OTUs than the other methods for the other data sets.

Correlation between the methods. Agreement between the methods was investigated by calculating Spearman's rank correlation between all pairs of output metrics (Fig 4). In the simulated data, FFMANOVA and ASCA had higher agreement than any other pair of methods, with correlations ranging from 0.6 to 0.75 . In addition, the correlations were generally higher for scenarios with many differentially abundant OTUs for all methods. The results from the experimental data sets also showed that FFMANOVA and ASCA had highest agreement, with correlations ranging from 0.4 to 0.9 . However, correlations varied considerably between the 
A) Simulations

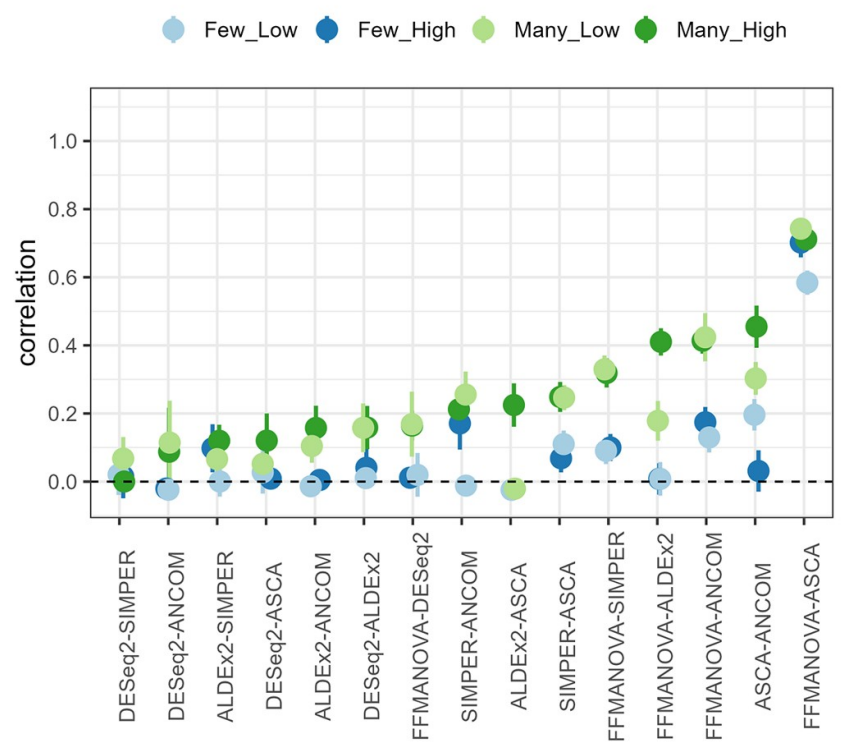

B) Experimental data

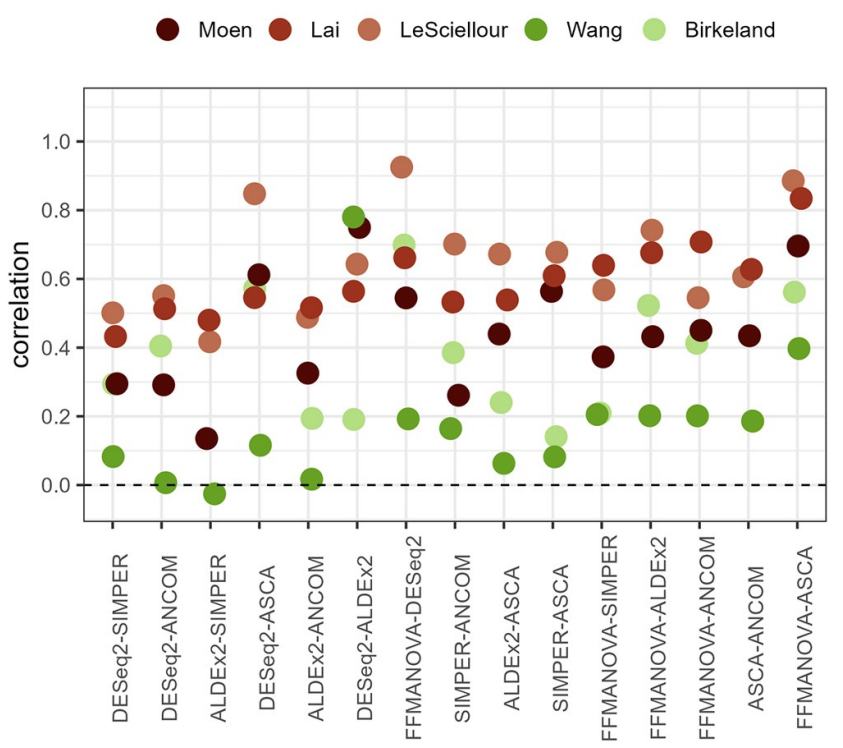

Fig 4. Spearman's correlation ( $\mathrm{Y}$-axis) calculated for pairwise comparison of statistical methods ( $\mathrm{X}$-axis) for (A) simulated data and (B) five experimental data sets. Each point represents Spearman's rank correlation coefficient between OTU ranking metrics from the two methods compared.

https://doi.org/10.1371/journal.pone.0259973.g004

data sets. Highest agreements were observed for the animal studies, which are more controlled, and where interindividual variation is smaller compared to studies involving human subjects. The Moen and Wang data sets had a lower correlation between the methods than the other data sets for many comparisons, which could be explained by the fact that there are more than two levels of the diet factor, and omnibus tests will therefore not completely agree with the pairwise comparisons.

Impact of OTU abundance. Gut microbiome data are typically represented by a few dominant OTUs (relative abundance $>1 \%$ ) and a majority of low-abundant OTUs. One is therefore interested in ranking OTUs independent of their average abundance to detect biologically relevant changes in low-abundant OTUs. For all methods, except SIMPER, correlations in the range 0.1-0.4 were observed between the ranking statistics and the (log) mean relative abundance. However, it was not consistent between the data sets which method gave higher correlations. Correlations between the relative abundance and the ranking statistics were highest for the Moen data set and lowest for the Birkeland data set (S2 Table). ANCOM differed from the other methods as the ranking of OTUs was highly dependent on the abundance (Fig 5 and S1 Fig). In particular, the results indicated that highly abundant OTUs had either very high or very low $\mathrm{W}$-stat, while low-abundant OTUs always had medium-to-low Wstat. To the best of our knowledge, this finding has not been reported before and shows that ANCOM is not able to identify changes in low-abundant OTUs. In the simulation study, clear differences between the scenarios with "Few" or "Many" differentially abundant OTUs can be observed (Fig 5A and 5C), and similar patterns are reflected for the experimental data (Fig 5B and 5D). It has been shown that power of ANCOM drops when the number of differentially abundant OTUs exceeds 25\% [28]. In our "Many" simulations, 50\% of the OTUs are differentially abundant, which justifies why ANCOM performed poorly for these scenarios. The dependency on the relative abundance can be more problematic also for the "Many" scenarios (Fig 5C) and data sets with many differentially abundant OTUs as, for instance, the Moen data set (Fig 5D). 
A) Simulation Few_Low

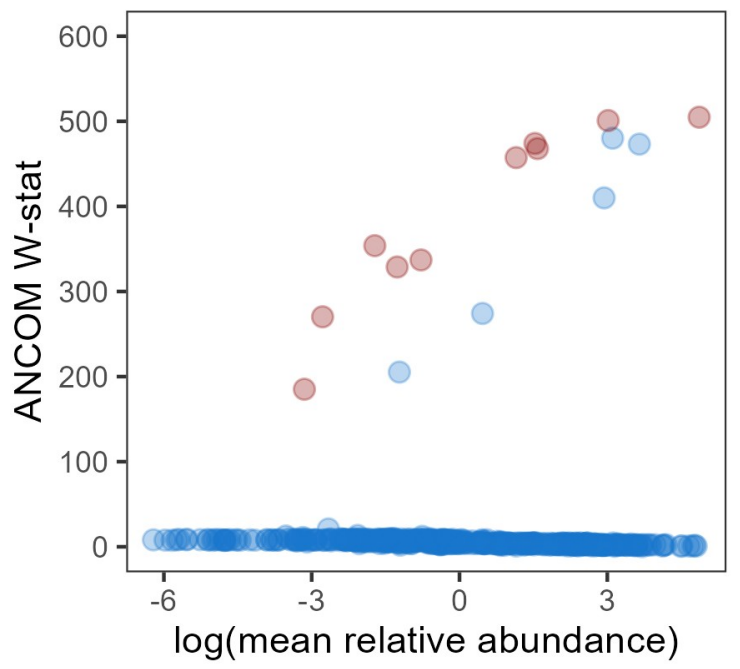

C) Simulation Many_Low

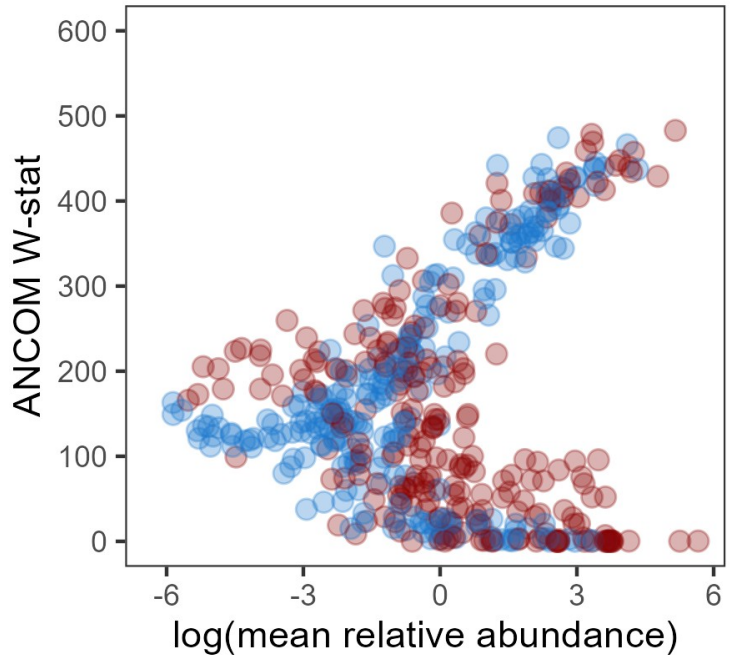

B) Birkeland data set

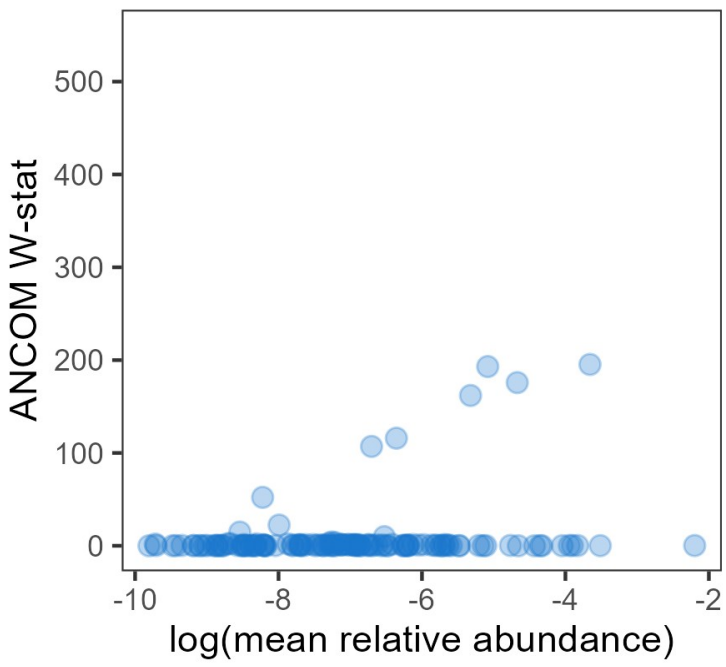

D) Moen data set

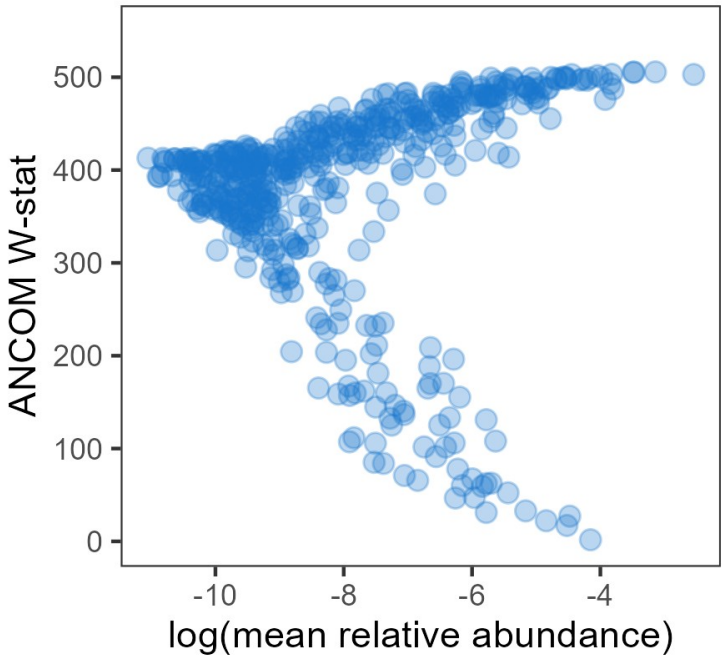

Fig 5. Mean relative abundance (log-scale) plotted versus ANCOM W-stat. (A) The "Few-Low" simulation scenario and (B) Birkeland data set, (C) "Many-Low" simulation scenario and (D) Moen data set. Red points (panels A and C) indicate differentially abundant OTUs.

https://doi.org/10.1371/journal.pone.0259973.g005

\section{Discussion}

Diet is considered to be an important driver of microbiome variation [51]. However, in observational population-based studies, diet consistently accounts for only a small proportion of microbiome variation, and this is partly due to large interindividual differences in microbiome composition, small sample sizes and limitations in study designs such as potentially insufficient washout periods in crossover studies [51-55]. In general, higher interindividual variation is observed in gut microbiome of human subjects compared to animal species [56]. This was confirmed by our results, and it can partly explain the lower diet related effect on the gut microbiomes in the two human studies. Variation in how much a diet can influence the microbiome is also dependent on the nutritional differences of the compared diets. Nevertheless, use 
of animal models to study a causal role of the gut microbiome in health and disease is an established practice although animal models lack the specific interactions present in the complex system of a human organism [57].

Univariate and multivariate analyses provide information at different levels. Biologists will often find that the outputs of univariate analyses are easier to interpret compared to those generated by multivariate analyses, though assumptions are similar for both method types [10]. In general, multivariate methods provide a more holistic overview of differences between samples and account for correlations and interactions between the variables, whereas univariate methods are well suited to point out the differences for specific microbial groups. Therefore, the two levels of analysis provide complementary information, and it is generally of biological interest to report differences at both levels.

FFMANOVA and ASCA consider the covariance between all OTUs. It could therefore be expected that these methods would be better at detecting scenarios with many differentially abundant OTUs due to the consistency at large phenomenon, i.e. that many OTUs carry the same information and such methods collectively are able to detect small effects that are not significant at the univariate level $[58,59]$. At the community level, these methods performed similarly to the distance-based methods. At the OTU level, they had considerably higher sensitivity to identify true positive OTUs than the other methods in the "Many" OTU scenarios.

FFMANOVA and ASCA depend on the relative scaling of the OTUs, while for the distance-based methods it depends on the chosen distance measure. It is a common practice in many areas to scale all variables to equal variance thus giving them an equal weight in the model, but other options are also possible, see, for instance, van den Berg et al. [60]. The clrtransformation puts the variables at comparable levels, and the need for scaling is less obvious. However, the highly abundant OTUs might still have slightly higher variance and scaling should be considered depending on the data characteristics and the biological interpretation. With scaling, all OTUs have the same contribution in the analysis, whereas without scaling the more abundant OTUs will dominate the analyses and the inferences will be related to the more abundant OTUs.

It is a known fact that microbial sequence data are zero-inflated, and rare OTUs should be removed prior to downstream statistical analyses. We have observed that the threshold for filtering out OTUs can significantly affect the results both at the community and OTU levels. This can be exemplified in the human Birkeland data set, where stricter OTU-filtering performed in the present study resulted in no significant treatment effect by ASCA, in contrast to the original publication [44].

The tools tested in the present study vary in flexibility. SIMPER allows only pairwise comparisons and ANOSIM provides multigroup analysis, but it is not suited for multifactorial study designs. The other methods can employ more complex models with multiple factors with varying number of levels, and corresponding interactions. In experiments with repeated measurements, the subject effect is often included as a random factor. Neither of the methods discussed here can do this, hence the subject effect was included as a fixed factor. These aspects should be considered when selecting methods because different study designs might require different types of statistical models and tests. Newer developments of ASCA, namely ASCA+ [61] and LiMM-PCA [62], increase flexibility when there are unbalanced designs or random (i.e. subject) effects, respectively. Even so, the longitudinal modelling of large microbiome data sets in combination with multiple covariates is only starting to emerge [6].

Although Spearman's rank correlation indicated good agreement for the animal studies (Fig 4), little overlap between lists of "significant" results could be detected (results not shown). There can be several reasons for this. One important aspect is that different criteria must be used to define "significance" or generally "importance". FFMANOVA, SIMPER, 
DESeq2 and ALDEx2 provide p-values, while more heuristic tools must be applied with ASCA and ANCOM. For some methods, such as FFMANOVA and ANCOM, the ranking is related to all levels of the experimental factors, whereas the other methods use only pairwise comparisons (SIMPER) or comparison against a selected control/reference level (ALDEx2 and DESeq2). ASCA provides a test related to all levels at the community level, while the PLS-DA step relates to pairwise comparisons at the OTU level. This will introduce a bias for data sets with multilevel factors where more than one level is different from each other. Nevertheless, we chose to compare these rankings as this is the output that is available to the user. In the simulations only one level of one factor was designed to have an effect, hence the "omnibus" and the "specific" tests are more directly comparable. In experiments with multilevel factors, additional information can be obtained by looking at the clr-difference between the groups of interest in addition to the ranking statistics.

Moreover, differences in sample collection, sample preparation and sequencing contribute to additional variability, which, in turn, affects the validity of the results [63] and complicates comparisons across studies with similar interventions.

Past benchmarking studies $[9,64,65]$ have reported varying results from different tools, which was also confirmed in our study. Currently, there is no consensus for the best existing tool for detecting differentially abundant microbial taxa, and there is no reason to believe that one single method is best in all cases. Based on our simulations, the generic multivariate tools, ASCA and FFMANOVA, performed best. We anticipate that these results will inform future studies with more complex settings where more than one factor has an effect or interactions between experimental factors are included in the model.

In addition to performance, ease of use is an important aspect when selecting the appropriate tool. FFMANOVA and ASCA are based on standard statistical tools, namely PCA and ANOVA. Some of the tools designed for microbiome high-throughput sequencing data, on the other hand, can be difficult for non-statisticians, and it can be questioned if the users are able to interpret all parameters correctly, even if the methods are supported by comprehensive documentation. It is always good scientific practice to compare and report outputs from several methods. There are no standards on how to report multiple modelling results, and there is a high risk of "fishing for significance" when several methods are applied [66]. Before designing the experiment, researchers should be aware of the different properties of the statistical methods and consider whether it is most important not to miss out on any possible findings or to obtain robust results. In the latter case, OTUs should be reported as differentially abundant only if they were flagged as "significant" by several methods [66].

\section{Conclusion}

In the present study, we compared the performance of several multivariate ANOVA-like statistical methods taking four simulated scenarios and five real dietary intervention microbiome data sets as examples. At the community level, all the different methods came to similar conclusions; at the OTU level, the agreement between the methods considerably varied. ANCOM provided output metrics that were dependent on the average abundance, making it impossible to detect differences in low-abundant OTUs. At the OTU level, the ranking of OTUs obtained with different methods correlated better for animal studies than for human studies, possibly due to lower interindividual variation in animal studies. Based on the simulation results we advise applying FFMANOVA and ASCA for overall and pairwise comparisons of microbiome data, respectively, also because these methods provide output at both the community and OTU levels, can handle several design factors, as well as other data types common in microbiome research. 


\section{Methods}

\section{Experimental design and data characteristics}

The dietary intervention data sets were selected based on the study design, with a minimum of two independent variables (for example, diet and dose; see S1 Table for details). Prior to the statistical analyses, the data were filtered to keep the OTUs that were present: (1) with relative abundance more than $0.005 \%$ in an individual, and (2) in at least $50 \%$ of the individuals and in one of the groups. For each study in total 507, 561, 560, 397 and 216 OTUs passed this filter and were subsequently used in downstream analyses (S1 Table and S2 File). All statistical analyses were performed in R version 4.1.0 [67] unless otherwise specified and were run in 999 permutations.

Simulated data were generated using the R package metaSPARSim [68] developed for simulating $16 \mathrm{~S}$ rDNA data. The simulation was a two-step process: (1) modelling the abundance (expected counts for each experimental group) using a Gamma distribution; (2) modelling the within-group variability using a Multivariate Hypergeometric (MHG) distribution taking the output from step 1 as input parameters for the distribution. In addition, metaSPARSim contains functions for parameter estimation from observed data. We used data set 1 [42] (S1 Table) to estimate real starting parameters for the simulations. Raw counts were generated for the same number of OTUs as in the experimental data set, and subsequently pre-processed and analysed in the same manner as for the experimental data (described below). Four different scenarios were simulated, with a varying number of the differentially abundant OTUs ("Few" versus "Many") and the effect sizes ("Low" versus "High"). 100 data sets were generated and analysed for each of the four scenarios:

1. "Few - Low": 10 randomly selected OTUs were assigned random $\log 2$ fold changes from a uniform distribution with boundaries [3,4].

2. "Few-High": 10 randomly selected OTUs were assigned random $\log 2$ fold changes from a uniform distribution with boundaries [8,9].

3. "Many - Low": 254 randomly selected OTUs were assigned random $\log 2$ fold changes from a uniform distribution with boundaries $[3,4]$.

4. "Many-High": 254 randomly selected OTUs were assigned random log2 fold changes from a uniform distribution with boundaries $[8,9]$.

Zero-value replacements were done prior to clr-transformation $[27,69]$ by applying function cMultRepl with a setting method = 'CZM' in the R package zCompositions [70]. Zero replacement is an ongoing and yet unsolved statistical problem in microbiome research, and newer methods are constantly being developed and applied to both simulated and experimental data sets [71-74].

ANOSIM and PERMANOVA were run using the functions anosim and adonis in the R package vegan [75], with a setting method = Euclidean and the clr-transformed data as input.

SIMPER was run on filtered relative abundance data using function simper from the $\mathrm{R}$ package vegan [75]. The $\mathrm{p}$-values for each OTU between selected pairwise comparisons of diet levels were used as a ranking metric; the p-value represents the probability of getting a larger contribution to the Bray-Curtis dissimilarity in a random permutation of the group factor.

FFMANOVA was performed by using the function ffmanova implemented in the R package ffmanova [35] on the clr-transformed and standardised data. Raw p-values were used as a ranking metric.

ASCA was run on the clr-transformed and centred data using an in-house implementation in MATLAB (R2018b, The MathWorks Inc.); p-values at the community level were calculated 
by permutation tests $(n=999)$. PLS-DA models [76] with the ASCA diet effect matrix, residuals as a predictor and factor levels as a response were used for pairwise comparison of diet levels. Variable Importance in Prediction (VIP) values [76] were used to identify significant OTUs, and the VIP threshold was set using the Uninformative Variable Elimination (UVE) method [77]. The UVE procedure was repeated 100 times, and OTUs that were above the threshold in $>95 \%$ of the repetitions were defined as significant. For study designs with two diet levels, the ASCA loadings were used to rank OTUs, while PLS-DA regression coefficients were used for pairwise comparisons of multilevel diet factors.

ANCOM was run by using the ANCOM 2.0 source code implemented in R [28] using filtered relative abundance data as input. The $\mathrm{W}$-stat was used to rank OTUs indicating the number of significantly different pairwise log-ratios while adjusting for FDR by applying a Benjamini-Hochberg correction at a 0.05 level of significance.

ALDEx2 was run using the functions aldex.clr and aldex.glm from the R package ALDEx2 v.1.18.0 [26]. We used raw counts as input and p-values for selected factor level contrasts to rank OTUs.

DESeq2 was run using the functions DESeqDataSetFromMatrix (to generate object), DESeq (for analysis) and results (to extract results) from the Bioconductor package version 1.32.0 [30]. We used raw counts as input and raw p-values for selected level contrasts to rank OTUs (default settings).

\section{Factor level comparisons}

For study designs with more than two diet groups, only one pairwise comparison was analysed for the methods based on the pairwise group comparison, namely ALDEx2, ASCA and SIMPER. The following pairs with the most contrasting outcomes were compared: (1) BSG group vs. IN group [42]; (2) control group vs. 3 g HMW [50] and (3) Placebo 6 weeks group vs. Fibre 6 weeks group [44].

Differentially abundant OTUs were identified by setting thresholds on the ranking metrics for each method. For the methods providing p-values, the threshold was set to 0.01 . For ANCOM, the $60^{\text {th }}$ percentile of the empirical distribution of the $\mathrm{W}$-stat was used as a threshold. For ASCA, OTUs selected in 95 out of 100 UVE-runs were identified as differentially abundant.

True Positive Rate (TPR) and True Negative Rate (TNR) were calculated for the simulated data. TPR (also called Power or Sensitivity) is calculated as TP/P, where TP is the number of true differentially abundant OTUs identified by a statistical method and $P$ is the number of differentially abundant OTUs defined in the simulation setup. The TNR (also called Specificity) is calculated as $T N / N$, where $T N$ is the number of true non-differentially abundant OTUs identified by a statistical method and $N$ is the corresponding number defined in the simulation setup.

\section{Supporting information}

S1 Fig. Pairwise scatterplots between OTU ranking metrics for each of the statistical methods. The (A) Moen, (B) Lai, (C) Le Sciellour, (D) Wang and (E) Birkeland data sets. (PDF)

S2 Fig. Explained variance for simulated data and the number of simulations where the simulated effect was detected. Differentially abundant OTUs are shown in red and non-differentially abundant OTUs in black.

(PDF)

S1 Table. An overview of the experimental studies and their data characteristics. (XLSX) 
S2 Table. Number and per cent of significant OTUs detected by each method. (XLSX)

S1 File. Summary table of the results obtained for the OTU level across five experimental data sets for the methods SIMPER, ASCA, FFMANOVA, ANCOM, ALDEx2 and DESeq2. (XLSX)

S2 File. Filtered relative abundance data and metadata for five experimental data sets. (XLSX)

S3 File. RData file with raw counts, relative abundance and clr-transformed and filtered data for the Moen data set. (RDATA)

S4 File. R script for the Moen data set analyses.

$(\mathrm{R})$

S5 File. R script for the simulated data analyses.

(R)

S6 File.

(DS_STORE)

S7 File.

(RHISTORY)

\section{Acknowledgments}

We thank all the authors who were involved in data generation used in the present study. All data sets are properly cited and referred to in S1 Table.

\section{Author Contributions}

Conceptualization: Ingrid Måge, Ida Rud, Ingunn Berget.

Data curation: Maryia Khomich.

Formal analysis: Maryia Khomich, Ingrid Måge, Ingunn Berget.

Funding acquisition: Ingrid Måge, Ida Rud.

Investigation: Maryia Khomich, Ingrid Måge, Ida Rud, Ingunn Berget.

Methodology: Maryia Khomich, Ingrid Måge, Ida Rud, Ingunn Berget.

Project administration: Maryia Khomich, Ingrid Måge, Ida Rud.

Resources: Ida Rud.

Software: Maryia Khomich, Ingrid Måge, Ingunn Berget.

Supervision: Ingrid Måge, Ida Rud, Ingunn Berget.

Validation: Maryia Khomich, Ingrid Måge, Ingunn Berget.

Visualization: Maryia Khomich, Ingrid Måge, Ida Rud, Ingunn Berget.

Writing - original draft: Maryia Khomich, Ingrid Måge, Ida Rud, Ingunn Berget.

Writing - review \& editing: Maryia Khomich, Ingrid Måge, Ida Rud, Ingunn Berget. 


\section{References}

1. Li H. Microbiome, metagenomics, and high-dimensional compositional data analysis. Annu Rev Stat Appl. 2015; 2:73-94. https://doi.org/10.1146/annurev-statistics-010814-020351.

2. Blanco-Míguez A, Fdez-Riverola $F$, Sánchez B, Lourenço $A$. Resources and tools for the high-throughput, multi-omic study of intestinal microbiota. Brief Bioinform. 2019; 20(3):1032-56. https://doi.org/10. 1093/bib/bbx156 PMID: 29186315

3. Knight R, Vrbanac A, Taylor BC, Aksenov A, Callewaert C, Debelius J, et al. Best practices for analysing microbiomes. Nat Rev Microbiol. 2018; 16(7):410-22. https://doi.org/10.1038/s41579-018-0029-9 PMID: 29795328

4. Gloor GB, Reid G. Compositional analysis: a valid approach to analyze microbiome high-throughput sequencing data. Can J Microbiol. 2016; 62(8):692-703. https://doi.org/10.1139/cjm-2015-0821 PMID: 27314511

5. Lê Cao K-A, Costello M-E, Lakis VA, Bartolo F, Chua X-Y, Brazeilles R, et al. MixMC: a multivariate statistical framework to gain insight into microbial communities. PLoS One. 2016; 11(8):e0160169. https:// doi.org/10.1371/journal.pone.0160169 PMID: 27513472

6. Mallick H, Ma S, Franzosa EA, Vatanen T, Morgan XC, Huttenhower C. Experimental design and quantitative analysis of microbial community multiomics. Genome Biol. 2017; 18(1):228. https://doi.org/10. 1186/s13059-017-1359-z PMID: 29187204

7. Waldron L. Data and statistical methods to analyze the human microbiome. mSystems. 2018; 3(2): e00194-17. https://doi.org/10.1128/mSystems.00194-17 PMID: 29556541

8. Schloss PD. Identifying and overcoming threats to reproducibility, replicability, robustness, and generalizability in microbiome research. mBio. 2018; 9(3):e00525-18. https://doi.org/10.1128/mBio.0052518 PMID: 29871915

9. Weiss S, Xu ZZ, Peddada S, Amir A, Bittinger K, Gonzalez A, et al. Normalization and microbial differential abundance strategies depend upon data characteristics. Microbiome. 2017; 5(1):27. https://doi. org/10.1186/s40168-017-0237-y PMID: 28253908

10. Paliy $\mathrm{O}$, Shankar V. Application of multivariate statistical techniques in microbial ecology. Mol Ecol. 2016; 25(5):1032-57. https://doi.org/10.1111/mec.13536 PMID: 26786791

11. Morton JT, Sanders J, Quinn RA, McDonald D, Gonzalez A, Vázquez-Baeza Y, et al. Balance trees reveal microbial niche differentiation. mSystems. 2017; 2(1):e00162-16. https://doi.org/10.1128/ mSystems.00162-16 PMID: 28144630

12. Wang S, Cai TT, Li H. Hypothesis testing for phylogenetic composition: a minimum-cost flow perspective. Biometrika. 2021; 108(1):17-36. https://doi.org/10.1093/biomet/asaa061 PMID: 33716568

13. Anderson MJ. A new method for non-parametric multivariate analysis of variance. Austral Ecol. 2001; 26(1):32-46. https://doi.org/10.1111/j.1442-9993.2001.01070.ppx.

14. Anderson MJ, Walsh DC. PERMANOVA, ANOSIM, and the Mantel test in the face of heterogeneous dispersions: what null hypothesis are you testing? Ecol Monogr. 2013; 83(4):557-74. https://doi.org/10. 1890/12-2010.1.

15. Clarke KR. Non-parametric multivariate analyses of changes in community structure. Aust J Ecol. 1993; 18(1):117-43. https://doi.org/10.1111/j.1442-9993.1993.tb00438.x.

16. Schroeder PJ, Jenkins DG. How robust are popular beta diversity indices to sampling error? Ecosphere. 2018; 9(2):e02100. https://doi.org/10.1002/ecs2.2100.

17. Wong RG, Wu JR, Gloor GB. Expanding the UniFrac toolbox. PLoS One. 2016; 11(9):e0161196. https://doi.org/10.1371/journal.pone.0161196 PMID: 27632205

18. Lozupone $C$, Knight R. UniFrac: a new phylogenetic method for comparing microbial communities. Appl Environ Microbiol. 2005; 71(12):8228-35. https://doi.org/10.1128/AEM.71.12.8228-8235.2005 PMID: 16332807

19. Lozupone C, Lladser ME, Knights D, Stombaugh J, Knight R. UniFrac: an effective distance metric for microbial community comparison. ISME J. 2011; 5(2):169-72. https://doi.org/10.1038/ismej.2010.133 PMID: 20827291

20. Roldán Ahumada JA, Avendaño Garrido ML. A commentary on diversity measures UniFrac in very small sample size. Evol Bioinform. 2019; 15:1176934319843515. https://doi.org/10.1177/ 1176934319843515 PMID: 31040637

21. Schloss PD. Evaluating different approaches that test whether microbial communities have the same structure. ISME J. 2008; 2(3):265-75. https://doi.org/10.1038/ismej.2008.5 PMID: 18239608

22. Warton $\mathrm{DI}$, Wright ST, Wang Y. Distance-based multivariate analyses confound location and dispersion effects. Methods Ecol Evol. 2012; 3(1):89-101. https://doi.org/10.1111/j.2041-210X.2011.00127.x. 
23. Randolph TW, Zhao S, Copeland W, Hullar M, Shojaie A. Kernel-penalized regression for analysis of microbiome data. Ann Appl Stat. 2018; 12(1):540-66. https://doi.org/10.1214/17-AOAS1102 PMID: 30224943

24. Tang Z-Z, Chen G, Alekseyenko AV. PERMANOVA-S: association test for microbial community composition that accommodates confounders and multiple distances. Bioinformatics. 2016; 32(17):261825. https://doi.org/10.1093/bioinformatics/btw311 PMID: 27197815

25. Wu C, Chen J, Kim J, Pan W. An adaptive association test for microbiome data. Genome Med. 2016; 8 (1):56. https://doi.org/10.1186/s13073-016-0302-3 PMID: 27198579

26. Fernandes AD, Reid JN, Macklaim JM, McMurrough TA, Edgell DR, Gloor GB. Unifying the analysis of high-throughput sequencing datasets: characterizing RNA-seq, 16S rRNA gene sequencing and selective growth experiments by compositional data analysis. Microbiome. 2014; 2(1):15. https://doi.org/10. 1186/2049-2618-2-15.

27. Gloor GB, Macklaim JM, Pawlowsky-Glahn V, Egozcue JJ. Microbiome datasets are compositional: and this is not optional. Front Microbiol. 2017; 8:2224. https://doi.org/10.3389/fmicb.2017.02224 PMID: 29187837

28. Mandal S, Van Treuren W, White RA, Eggesbø M, Knight R, Peddada SD. Analysis of composition of microbiomes: a novel method for studying microbial composition. Microb Ecol Health Dis. 2015; 26 (1):27663.

29. Robinson MD, McCarthy DJ, Smyth GK. edgeR: a Bioconductor package for differential expression analysis of digital gene expression data. Bioinformatics. 2010; 26(1):139-40. https://doi.org/10.1093/ bioinformatics/btp616 PMID: 19910308

30. Love MI, Huber W, Anders S. Moderated estimation of fold change and dispersion for RNA-seq data with DESeq2. Genome Biol. 2014; 15(12):1-21. https://doi.org/10.1186/s13059-014-0550-8 PMID: 25516281

31. Lin H, Peddada SD. Analysis of compositions of microbiomes with bias correction. Nat Commun. 2020; 11(1):1-11. https://doi.org/10.1038/s41467-019-13993-7 PMID: 31911652

32. Saccenti E, Hoefsloot HC, Smilde AK, Westerhuis JA, Hendriks MM. Reflections on univariate and multivariate analysis of metabolomics data. Metabolomics. 2014; 10(3):361-74. https://doi.org/10.1007/ s11306-013-0598-6.

33. Smilde AK, Jansen JJ, Hoefsloot HC, Lamers R-JA, Van Der Greef J, Timmerman ME. ANOVA-simultaneous component analysis (ASCA): a new tool for analyzing designed metabolomics data. Bioinformatics. 2005; 21(13):3043-8. https://doi.org/10.1093/bioinformatics/bti476 PMID: 15890747

34. Stahle L, Wold S. Multivariate analysis of variance (MANOVA). Chemometr Intell Lab 1990; 9:127-41. https://doi.org/10.1016/0169-7439(90)80094-M.

35. Langsrud Ø. 50-50 multivariate analysis of variance for collinear responses. J R Stat Soc-Ser D Stat. 2002; 51(3):305-17. https://doi.org/10.1111/1467-9884.00320.

36. Langsrud $\varnothing$. Rotation tests. Stat Comput. 2005; 15(1):53-60. https://link.springer.com/content/pdf/10. 1007/s11222-005-4789-5.pdf.

37. Combrink M, Du Preez I, Ronacher K, WalzI G, Loots DT. Time-dependent changes in urinary metabolome before and after intensive phase tuberculosis therapy: a pharmacometabolomics study. OMICS. 2019; 23(11):560-72. https://doi.org/10.1089/omi.2019.0140 PMID: 31613685

38. Gómez-Canela C, Prats E, Lacorte S, Raldúa D, Piña B, Tauler R. Metabolomic changes induced by nicotine in adult zebrafish skeletal muscle. Ecotox Environ Safe. 2018; 164:388-97. https://doi.org/10 1016/j.ecoenv.2018.08.042 PMID: 30142605

39. Trimigno A, Khakimov B, Savorani F, Tenori L, Hendrixson V, Čivilis A, et al. Investigation of Variations in the Human Urine Metabolome amongst European Populations: An Exploratory Search for Biomarkers of People at Risk-of-Poverty. Mol Nutr Food Res. 2019; 63(1):1800216. https://doi.org/10.1002/ mnfr.201800216 PMID: 29757492

40. Bjerke GA, Rudi K, Avershina E, Moen B, Blom H, Axelsson L. Exploring the brine microbiota of a traditional Norwegian fermented fish product (Rakfisk) from six different producers during two consecutive seasonal productions. Foods. 2019; 8(2):72. https://doi.org/10.3390/foods8020072 PMID: 30769832

41. Måge I, Steppeler C, Berget I, Paulsen JE, Rud I. Multi-way methods for understanding longitudinal intervention effects on bacterial communities. bioRxiv: 363630v1 [Preprint]. 2018:[cited 1 Sept 2021]. Available from: https://www.biorxiv.org/content/10.1101/363630v1.

42. Moen B, Henjum K, Måge I, Knutsen SH, Rud I, Hetland RB, et al. Effect of dietary fibers on cecal microbiota and intestinal tumorigenesis in azoxymethane treated A/J Min/+ mice. PLoS One. 2016; 11(5): e0155402. https://doi.org/10.1371/journal.pone.0155402 PMID: 27196124

43. Moen B, Røssvoll E, Måge I, Møretrø T, Langsrud S. Microbiota formed on attached stainless steel coupons correlates with the natural biofilm of the sink surface in domestic kitchens. Can J Microbiol. 2016; 62(2):148-60. https://doi.org/10.1139/cjm-2015-0562 PMID: 26758935 
44. Birkeland E, Gharagozlian S, Birkeland KI, Valeur J, Måge I, Rud I, et al. Prebiotic effect of inulin-type fructans on faecal microbiota and short-chain fatty acids in type 2 diabetes: a randomised controlled trial. Eur J Nutr. 2020; 59:3325-38. https://doi.org/10.1007/s00394-020-02282-5 PMID: 32440730

45. Segata N, Izard J, Waldron L, Gevers D, Miropolsky L, Garrett WS, et al. Metagenomic biomarker discovery and explanation. Genome Biol. 2011; 12(6):R60. https://doi.org/10.1186/gb-2011-12-6-r60 PMID: 21702898

46. Chi C, Xue Y, Lv N, Hao Y, Liu R, Wang Y, et al. Longitudinal gut bacterial colonization and its influencing factors of low birth weight infants during the first 3 months of life. Front Microbiol. 2019; 10:1105. https://doi.org/10.3389/fmicb.2019.01105 PMID: 31156608

47. Girard C, Tromas N, Amyot M, Shapiro BJ. Gut microbiome of the canadian arctic Inuit. mSphere. 2017; 2(1):e00297-16. https://doi.org/10.1128/mSphere.00297-16 PMID: 28070563

48. Lai Z-L, Tseng C-H, Ho HJ, Cheung CK, Lin J-Y, Chen Y-J, et al. Fecal microbiota transplantation confers beneficial metabolic effects of diet and exercise on diet-induced obese mice. Sci Rep. 2018; 8 (1):15625. https://doi.org/10.1038/s41598-018-33893-y PMID: 30353027

49. Le Sciellour M, Labussière E, Zemb O, Renaudeau D. Effect of dietary fiber content on nutrient digestibility and fecal microbiota composition in growing-finishing pigs. PLoS One. 2018; 13(10):e0206159. https://doi.org/10.1371/journal.pone.0206159 PMID: 30356293

50. Wang Y, Ames NP, Tun HM, Tosh SM, Jones PJ, Khafipour E. High molecular weight barley $\beta$-glucan alters gut microbiota toward reduced cardiovascular disease risk. Front Microbiol. 2016; 7:129. https:// doi.org/10.3389/fmicb.2016.00129 PMID: 26904005

51. Vangay $P$, Johnson $A J$, Ward $T L, A l-G h a l i t h ~ G A$, Shields-Cutler RR, Hillmann $B M$, et al. US immigration westernizes the human gut microbiome. Cell. 2018; 175(4):962-72.e10. https://doi.org/10.1016/j.cell. 2018.10.029 PMID: 30388453

52. Hughes RL, Kable ME, Marco M, Keim NL. The role of the gut microbiome in predicting response to diet and the development of precision nutrition models. Part II: results. Adv Nutr. 2019; 10(6):979-98. https://doi.org/10.1093/advances/nmz049 PMID: 31225587

53. Johnson AJ, Vangay P, Al-Ghalith GA, Hillmann BM, Ward TL, Shields-Cutler RR, et al. Daily sampling reveals personalized diet-microbiome associations in humans. Cell Host Microbe. 2019; 25(6):789802. e5. https://doi.org/10.1016/j.chom.2019.05.005 PMID: 31194939

54. Rothschild D, Weissbrod O, Barkan E, Kurilshikov A, Korem T, Zeevi D, et al. Environment dominates over host genetics in shaping human gut microbiota. Nature. 2018; 555(7695):210-5. https://doi.org/10 1038/nature25973 PMID: 29489753

55. So D, Whelan K, Rossi M, Morrison M, Holtmann G, Kelly JT, et al. Dietary fiber intervention on gut microbiota composition in healthy adults: a systematic review and meta-analysis. Am J Clin Nutr. 2018; 107(6):965-83. https://doi.org/10.1093/ajcn/nqy041 PMID: 29757343

56. Nagpal R, Wang S, Solberg Woods LC, Seshie O, Chung ST, Shively CA, et al. Comparative microbiome signatures and short-chain fatty acids in mouse, rat, non-human primate, and human feces. Front Microbiol. 2018; 9:2897. https://doi.org/10.3389/fmicb.2018.02897 PMID: 30555441

57. Baker DH. Animal models in nutrition research. Nutr J. 2008; 138(2):391-6. https://doi.org/10.1093/jn/ 138.2.391 PMID: 18203909

58. Hui BS, Wold HOA. Consistency and consistency at large of partial least squares estimates. In: Jöreskog KG, Wold HOA, editors. Systems under indirect observation, part II. Amsterdam: North-Holland Publ. Co.; 1982. p. 119-30.

59. Schneeweiss $\mathrm{H}$. Consistency at large in models with latent variables. In: Haagen $\mathrm{K}$, Bartholomew DJ, Deistler M, editors. Statistical modelling and latent variables. Amsterdam: Elsevier; 1993. p. 299-320.

60. van den Berg RA, Hoefsloot HC, Westerhuis JA, Smilde AK, van der Werf MJ. Centering, scaling, and transformations: improving the biological information content of metabolomics data. BMC Genomics. 2006; 7(1):142. https://doi.org/10.1186/1471-2164-7-142.

61. Thiel M, Féraud B, Govaerts B. ASCA+ and APCA+: Extensions of ASCA and APCA in the analysis of unbalanced multifactorial designs. J Chemom. 2017; 31(6):e2895. https://doi.org/10.1002/cem.2895.

62. Martin M, Govaerts B. LiMM-PCA: Combining ASCA+ and linear mixed models to analyse high-dimensional designed data. J Chemom. 2020; 34(6):e3232. https://doi.org/10.1002/cem.3232.

63. Hughes RL, Marco ML, Hughes JP, Keim NL, Kable ME. The role of the gut microbiome in predicting response to diet and the development of precision nutrition models-Part I: overview of current methods. Adv Nutr. 2019; 10(6):953-78. https://doi.org/10.1093/advances/nmz022 PMID: 31225589

64. Hawinkel S, Mattiello F, Bijnens L, Thas O. A broken promise: microbiome differential abundance methods do not control the false discovery rate. Brief Bioinform. 2019; 20(1):210-21. https://doi.org/10.1093/ bib/bbx104 PMID: 28968702 
65. Thorsen J, Brejnrod A, Mortensen M, Rasmussen MA, Stokholm J, Al-Soud WA, et al. Large-scale benchmarking reveals false discoveries and count transformation sensitivity in 16S rRNA gene amplicon data analysis methods used in microbiome studies. Microbiome. 2016; 4(1):62. https://doi.org/10. 1186/s40168-016-0208-8 PMID: 27884206

66. Boulesteix AL, Binder H, Abrahamowicz M, Sauerbrei W. On the necessity and design of studies comparing statistical methods. Biometrical J. 2017; 60(1):216-8. https://doi.org/10.1002/bimj.201700129 PMID: 29193206

67. R Development Core Team. R: A language and environment for statistical computing. R Foundation for Statistical Computing, Vienna, Austria. https://www.R-project.org/. 2021.

68. Patuzzi I, Baruzzo G, Losasso C, Ricci A, Di Camillo B. metaSPARSim: a 16S rRNA gene sequencing count data simulator. BMC Bioinform. 2019; 20(9):1-13. https://doi.org/10.1186/s12859-019-2882-6 PMID: 31757204

69. Aitchison J. The analysis of compositional data. London: Chapman and Hall; 1986. 416 p.

70. Palarea-Albaladejo J, Martín-Fernández JA. zCompositions-R package for multivariate imputation of left-censored data under a compositional approach. Chemometr Intell Lab. 2015; 143:85-96. https:// doi.org/10.1016/j.chemolab.2015.02.019.

71. Brill B, Amir A, Heller R. Testing for differential abundance in compositional counts data, with application to microbiome studies. arXiv:190408937 [Preprint]. 2019:[cited 1 Sept 2021]. https://arxiv.org/pdf/1904. 08937.pdf.

72. Costea PI, Zeller G, Sunagawa S, Bork P. A fair comparison. Nat Methods. 2014; 11(4):359. https://doi. org/10.1038/nmeth.2897 PMID: 24681719

73. Wang S. Robust differential abundance test in compositional data. arXiv: 210108765 [Preprint]. 2021: [cited 1 Sept 2021]. https://arxiv.org/pdf/101.08765.pdf.

74. Lubbe S, Filzmoser P, Templ M. Comparison of zero replacement strategies for compositional data with large numbers of zeros. Chemometr Intell Lab. 2021;210:104248. https://doi.org/10.1016/j.chemolab. 2021.104248

75. Oksanen J, Blanchet FG, Friendly M, Kindt R, Legendre P, McGlinn D, et al. vegan: Community ecology package (R package version 2.5-5) https://cran.r-project.org/ and https://github.com/vegandevs/ vegan/. 2019.

76. Eriksson L, Byrne T, Johansson E, Trygg J, Vikström C. Multi-and megavariate data analysis basic principles and applications. Malmö, Sweden: Umetrics Academy; 2013.

77. Centner V, Massart D-L, de Noord OE, de Jong S, Vandeginste BM, Sterna C. Elimination of uninformative variables for multivariate calibration. Anal Chem. 1996; 68(21):3851-8. https://doi.org/10.1021/ ac960321m PMID: 21619260 